\title{
Response to everolimus is seen in TSC-associated SEGAs and angiomyolipomas independent of mutation type and site in TSC1 and TSC2
}

\author{
David J Kwiatkowski ${ }^{\star,}$, Michael R Palmer ${ }^{2}$, Sergiusz Jozwiak ${ }^{3}$, John Bissler ${ }^{4}$, David Franz ${ }^{5}$, Scott Segal ${ }^{6}$, \\ David Chen ${ }^{6}$ and Julian R Sampson ${ }^{7}$
}

Tuberous sclerosis complex is an autosomal dominant disorder that occurs owing to inactivating mutations in either TSC1 or TSC2. Tuberous sclerosis complex-related tumors in the brain, such as subependymal giant cell astrocytoma, and in the kidney, such as angiomyolipoma, can cause significant morbidity and mortality. Recently, randomized clinical trials (EXIST-1 and EXIST-2) of everolimus for each of these tuberous sclerosis complex-associated tumors demonstrated the benefit of this drug, which blocks activated mammalian target of rapamycin complex 1 . Here we report on the spectrum of mutations seen in patients treated during these trials and the association between mutation and response. TSC2 mutations were predominant among patients in both trials and were present in nearly all subjects with angiomyolipoma in whom a mutation was identified (97\%), whereas TSC1 mutations were rare in those subjects (3\%). The spectrum of mutations seen in each gene was similar to those previously reported. In both trials, there was no apparent association between mutation type or location within each gene and response to everolimus. Everolimus responses were also seen at a similar frequency for the $16-18 \%$ of patients in each trial in whom no mutation in either gene was identified. These observations confirm the strong association between TSC 2 mutation and angiomyolipoma burden seen in previous studies, and they indicate that everolimus response occurs regardless of mutation type or location or when no mutation in TSC1 or TSC2 has been identified.

European Journal of Human Genetics (2015) 23, 1665-1672; doi:10.1038/ejhg.2015.47; published online 18 March 2015

\section{INTRODUCTION}

Tuberous sclerosis complex (TSC) is an autosomal dominant disorder that occurs as a result of inactivating mutations in either TSC1 or TSC2, and affects about 1 in 10000 individuals worldwide. ${ }^{1}$ It is characterized by prominent neurodevelopmental features and by tumors that develop in the brain, skin, heart, kidneys and lungs. ${ }^{1}$

The brain tumor seen most commonly in TSC is the subependymal giant cell astrocytoma (SEGA), which is usually located near the foramen of Monro and develops in as many as $20 \%$ of individuals with TSC. $^{2}$ When they grow to be of significant size, SEGAs cause significant morbidity through ventricular obstruction, leading to hydrocephalus and compression of nearby brain structures. ${ }^{2,3}$ In the past, surgical resection had been the main treatment approach for these tumors, but both postoperative morbidity and recurrence after surgery are important clinical issues; this has led to the use of rapamycin and/or everolimus for the treatment of SEGAs. ${ }^{2,4-9}$

Renal angiomyolipomas are seen in about $80 \%$ of individuals with TSC; they cause the largest share of adult deaths from TSC, through increased risk of hemorrhage and loss of functional renal parenchyma leading to chronic kidney failure. ${ }^{10-12}$ Both surgical resection and therapeutic embolism have been used to control these tumors, and nephron-sparing approaches are considered mandatory.
More than 2000 nonsynonymous mutations have been identified in the TSC1 and TSC2 genes (http://chromium.liacs.nl/LOVD2/TSC/ home.php). ${ }^{13-32}$ In both TSC1 and TSC2, 50-60\% of all mutations are single base substitution mutations, and a large fraction of those are $\mathrm{C}$ to $\mathrm{T}$ transitions at $\mathrm{CpG}$ sites - likely due to deamination of a methylated $\mathrm{C}$ residue. In TSC1 the majority of the $\mathrm{C}$ to $\mathrm{T}$ transitions cause nonsense mutations, whereas in TSC2 both nonsense and missense mutations occur owing to this mechanism. Insertion and deletion mutations of size 1-4 nucleotides are also quite common in both TSC1 and TSC2, and usually occur at sites of short repetitive sequences. Large genomic deletions and rearrangements in the TSC2 gene are also relatively common and are seen in about $6 \%$ of unselected TSC patients. ${ }^{30}$ In contrast, large genomic deletions are quite rare in TSC1 and are seen in only about $0.5 \%$ of unselected cases. ${ }^{1,30}$ This may be due, in part, to a sequence within the adjacent $P K D 1$ gene that blocks the replication fork and could lead to doublestrand breaks in this region of chromosome $16^{34}$. Ten to fifteen percent of TSC patients have no mutation identified in these genes, despite a careful search, and it seems likely that mosaicism accounts for a significant fraction of those without an identified mutation.

A summation of published reports on mutation identification in $\mathrm{TSC}^{13-32}$ indicates that among patients with identified mutations, about $21 \%$ occur in TSC1 and 79\% occur in TSC2. ${ }^{33}$ This difference in mutation frequency in the two genes is likely due, in part, to the

${ }^{1}$ Brigham and Women's Hospital, Boston, MA, USA; ${ }^{2}$ Novartis Oncology Translational Medicine, Cambridge, MA, USA; ${ }^{3}$ The Children's Memorial Health Institute, Warsaw, Poland; ${ }^{4}$ St Jude Children's Research Hospital, University of Tennessee Health Science Center, Memphis, TN, USA; ${ }^{5}$ Cincinnati Children's Hospital Medical Center, Cincinnati, $\mathrm{OH}$, USA; ${ }^{6}$ Novartis Pharmaceuticals Corporation, East Hanover, NJ, USA; ${ }^{7}$ Institute of Medical Genetics, School of Medicine, Cardiff University, Cardiff, UK

*Correspondence: Dr DJ Kwiatkowski, Department of Medicine, Brigham and Women's Hospital, 1 Blackfan Circle, Boston, MA 02115 , USA. Tel: +1 617 3559005 ; Fax: +1 617355 9016; E-mail: dk@rics.bwh.harvard.edu

Received 16 October 2014; revised 17 January 2015; accepted 27 January 2015; published online 18 March 2015 
larger size of the coding region of TSC2 $(5.4 \mathrm{~kb})$ compared with TSC1 $(3.5 \mathrm{~kb})$, and it also appears to be due to an intrinsic difference in mutation rate given that about two-thirds of all cases of TSC are a result of new mutations.

EXIST-1 and EXIST-2 were multicenter, randomized, placebocontrolled clinical trials examining the benefit of everolimus for the treatment of SEGAs and angiomyolipoma, respectively. ${ }^{34,35}$ Subjects with SEGAs in EXIST-1 also had to have a diagnosis of TSC, whereas those with angiomyolipoma in EXIST-2 could have either TSC or sporadic lymphangioleiomyomatosis (LAM). In this article, we report on an analysis of TSC1 and TSC2 mutations in the subjects participating in the two trials.

\section{MATERIALS AND METHODS}

Patient recruitment and clinical results of the EXIST trials

Detailed reports on patient recruitment and conduct of the EXIST trials have been published elsewhere. ${ }^{34,35}$

\section{Mutation analysis}

Venous blood was collected in EDTA tubes from all patients (who had given informed consent) and sent to Quest Diagnostics (San Juan Capistrano, CA, USA) for genomic DNA extraction using the Gentra Systems Autopure LS (Minneapolis, MN, USA). Full exonic sequencing for TSC1 and TSC2 was performed on samples from the EXIST-1 trial at Novartis Pharmaceuticals Corporation Inc. (Cambridge, MA, USA). Amplification was performed using Advantage HF 2 PCR Kit (Clontech Laboratories Inc., Mountain View, CA, USA) with M13-labeled primers. Primer sequences are available upon request from MRP (michaelr.palmer@novartis.com).

PCR purification and standard M13-primered bidirectional Sanger sequencing was performed at GeneWiz Inc. (Cambridge, MA, USA). Sequence analysis was performed at Novartis Pharmaceuticals Corporation Inc., using Mutation Surveyor (SoftGenetics LLC, State College, PA, USA), with independent read confirmation. TSC1 and TSC2 duplication and/or deletion analysis was performed at Novartis Pharmaceuticals Corporation Inc., using MRC-Holland's P124 TSC1 and P046-B2 TSC2 MLPA Kits (MRC-Holland, Amsterdam, The Netherlands), according to the manufacturer's instructions with $100 \mathrm{ng}$ of DNA input. MLPA analysis was performed using GeneMarker Software (SoftGenetics LLC) with its MLPA analysis package. TSC2 exon 7 was excluded from analysis owing to high levels of variability in its amplification peak relative to other probe sets. A DNA sample was required to have at least two consecutive probe sets in the duplication and/or deletion range to be considered positive. All samples were run two times for confirmation. Sequencing and duplication/ deletion analysis for the EXIST-2 trial was performed using very similar PCR conditions and assays at Athena Diagnostics Inc. (Worcester, MA, USA), using its Complete Tuberous Sclerosis Evaluation panel. This analysis consists of Sanger sequencing of all exons of TSC1 and TSC2, and the same MLPA duplication and deletion assays for TSC1 and TSC2 described above.

\section{Mutation definition and assessment of pathogenicity}

The term mutation is used here to mean a sequence variant that is known or thought to ablate the function of the TSC1 or TSC2 gene transcript or protein product. We used several criteria to assess whether sequence variants were mutations. First, chain-terminating (nonsense and out-of-frame indels) and splice variants affecting consensus nucleotides were considered mutations. Second, missense and in-frame deletion variants were compared with those reported in the Leiden Open Variation Database (LOVD) for Tuberous Sclerosis Complex (http://chromium.liacs.nl/LOVD2/TSC/home.php), and information indicative of likely pathogenic significance was used to confer mutation status, when available. Third, in the remaining missense variant cases, the amino-acid Block Substitution Matrix ${ }^{36}$ was used to assess the importance of missense changes on function. Missense variants with a score $\leq-1$ were considered mutations.

\section{Nomenclature and database information}

Exon numbering for TSC1 and TSC2 was according to that used by the LOVD (http://chromium.liacs.nl/LOVD2/TSC). All variant data were submitted to that database.

\section{Statistical analysis}

Using GraphPad software (http://graphpad.com/quickcalcs), categorical variables were compared using the $\chi^{2}$ test for comparisons with an expected distribution and Fisher's exact test for comparisons between two sets of observations. $P$-values reported are nominal. No multiplicity adjustments were made, so statistical interpretation should be made with caution.

\section{RESULTS}

Mutation findings in the EXIST-1 and EXIST-2 trials

In the EXIST-1 trial, 117 patients with SEGA and TSC were randomly assigned to everolimus $(n=78)$ or placebo $(n=39) .{ }^{34}$ The median age of subjects was 9.5 years (range $0.8-26.6$ years). Twenty-seven $(35 \%)$ of 78 patients in the everolimus group and zero of 39 in the placebo group had a response in terms of a reduction in the total SEGA volume of $\geq 50 \%$. $^{34}$

DNA samples from 116 patients were available for mutation analysis; 97 of 116 (84\%) samples were found to have a mutation in either TSC1 or TSC2 (Table 1 and Supplementary Table 1). The distribution of mutation types was similar to that reported

Table 1 Mutation findings in EXIST-1: the SEGA trial

\begin{tabular}{|c|c|c|c|c|}
\hline Mutation type & TSC1 & TSC2 & Total & $\begin{array}{c}\text { Percentage of } 116, \\
\text { total number }\end{array}$ \\
\hline Deletion & 5 & 18 & 23 & 20 \\
\hline In-frame deletion/insertion & 0 & 3 & 3 & 3 \\
\hline Insertion & 3 & 2 & 5 & 4 \\
\hline Large deletion & 0 & 11 & 11 & 9 \\
\hline Missense & 0 & 13 & 13 & 11 \\
\hline Nonsense & 5 & 22 & 27 & 23 \\
\hline Splice & 0 & 15 & 15 & 13 \\
\hline Any & 13 & 84 & 97 & 84 \\
\hline No mutation identified & & & 19 & 16 \\
\hline Total & & & 116 & \\
\hline $\begin{array}{l}\text { Percentage of } 97 \text { subjects } \\
\text { with mutation identified }\end{array}$ & 13 & 87 & & \\
\hline
\end{tabular}

Table 2 Mutation findings in EXIST-2: the angiomyolipoma trial

\begin{tabular}{|c|c|c|c|c|}
\hline Mutation type & TSC1 & TSC2 & Total & $\begin{array}{c}\text { Percentage of } 109, \\
\text { total number }\end{array}$ \\
\hline Deletion & 1 & 11 & 12 & 11 \\
\hline In-frame deletion/insertion & 0 & 5 & 5 & 5 \\
\hline Insertion & 0 & 9 & 9 & 8 \\
\hline Large deletion & 1 & 6 & 7 & 6 \\
\hline Missense & 0 & 24 & 24 & 22 \\
\hline Nonsense & 0 & 25 & 25 & 23 \\
\hline Splice & 1 & 6 & 7 & 6 \\
\hline Any & 3 & 86 & 89 & 82 \\
\hline No mutation identified & & & 20 & 18 \\
\hline Total & & & 109 & \\
\hline $\begin{array}{l}\text { Percentage of } 89 \text { subjects } \\
\text { with mutation identified }\end{array}$ & 3 & 97 & & \\
\hline
\end{tabular}


previously. ${ }^{33}$ However, only $13(13 \%)$ of patients with defined mutations had mutations in TSC1, whereas 84 (87\%) had mutations in TSC2. This is a somewhat lower proportion of TSC1 mutations than was seen in other series, in which $21 \%$ had TSC1 mutations and $79 \%$ had TSC2 mutations $\left(P=0.0662, \chi^{2}\right.$ test). ${ }^{33}$ Nineteen of 116 $(16 \%)$ had no mutation identified; this was similar to multiple previous studies. ${ }^{13-32}$

In the EXIST-2 trial, 118 patients with angiomyolipoma, and TSC and/or LAM, were randomly assigned to receive everolimus $(n=79)$ or placebo $(n=39) .{ }^{35}$ The median age of subjects was 31 years (range 18.0-61.0 years); 78\% (92 of 118) of patients had angiomyolipomas in both kidneys, 29\% (34 of 118) had an angiomyolipoma of at least $8 \mathrm{~cm}$ in its longest dimension and nearly $40 \%$ (46 of 118) had a previous intervention, including $19 \%$ (22 of 118) with prior nephrectomy. Of patients receiving everolimus, $42 \%$ (33 of 79) showed a response at 12 weeks $v s \quad 0 \%$ ( 0 of 39) of those receiving placebo. ${ }^{35}$ Response was defined as a reduction in angiomyolipoma volume (sum of volumes of all target angiomyolipomas identified at

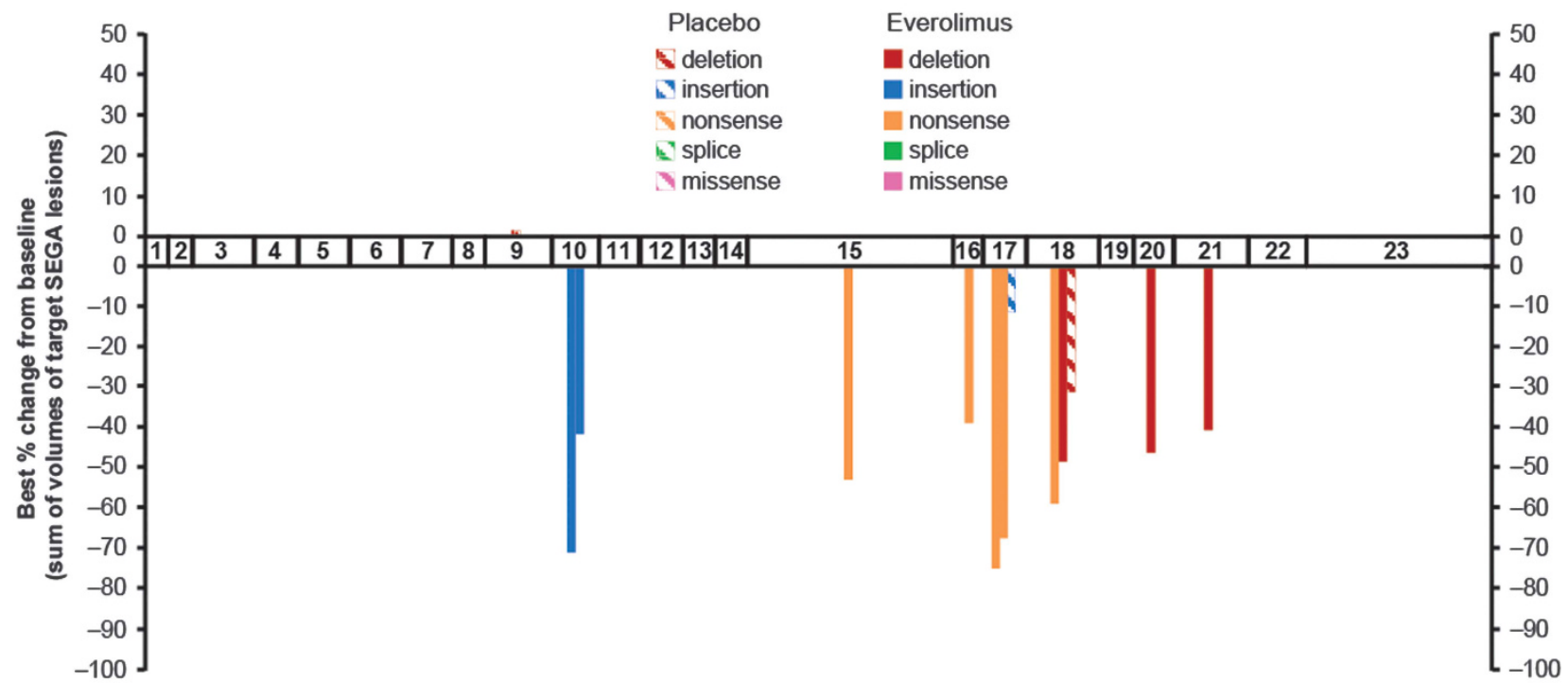

b

SEGA Response by TSC2 Gene Mutation

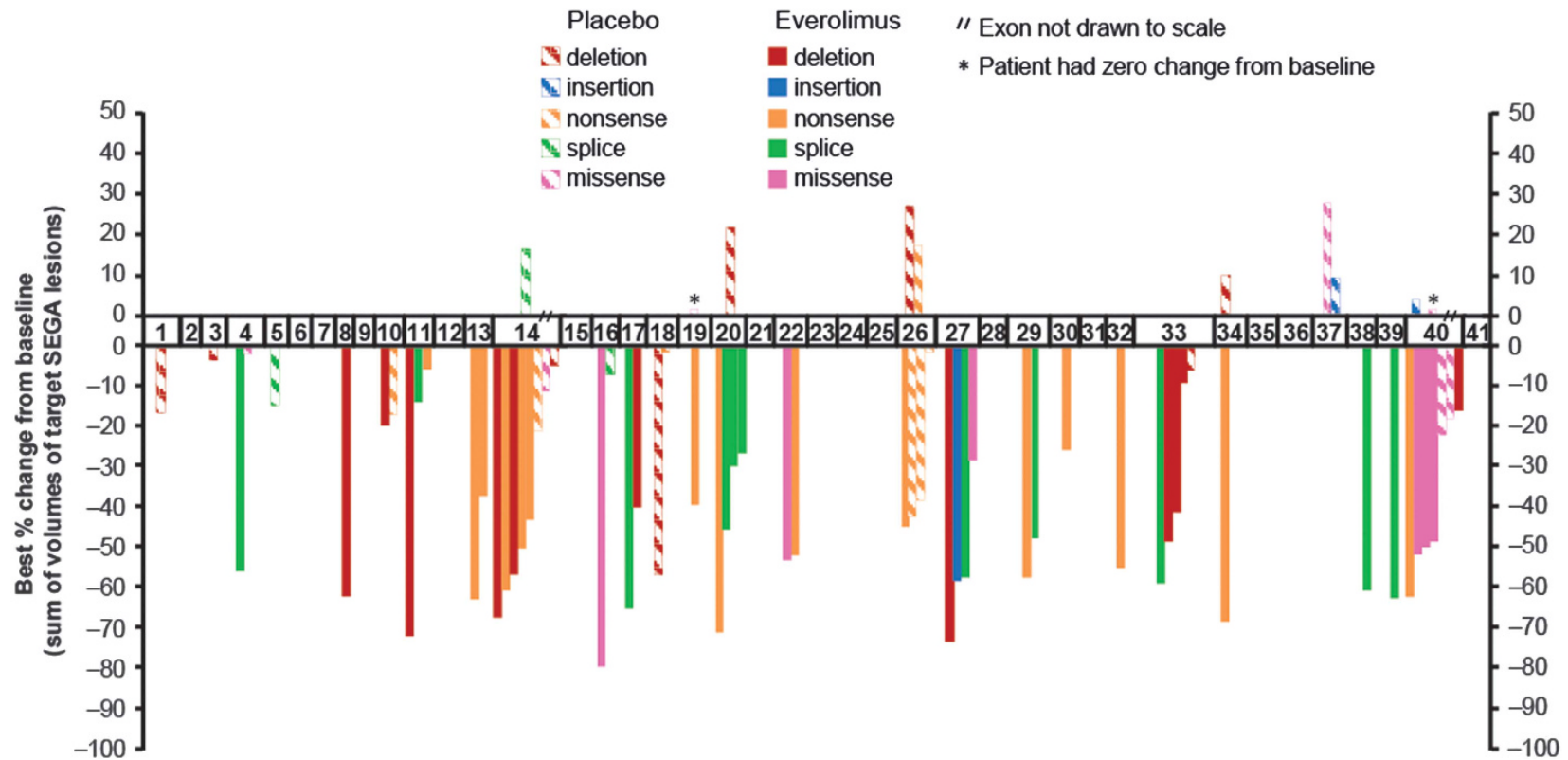

Figure 1 Clinical response to everolimus treatment of SEGAs in EXIST-1 according to mutation type and location in TSC1 and TSC2. The best percentage change in the sum of volumes of target SEGA lesions is shown on the $y$ axis. The mutation location and type is shown on the $x$ axis, which is a diagram of the exons (drawn proportional to size) of TSC1 (a) or TSC2 (b, c). Note that large deletions in TSC2 are indicated by their extent across the exons (c). Patients without an identified mutation are sorted by response (d). Subjects receiving placebo are shown with striped patterns, whereas those receiving everolimus are shown with solid patterns. 
baseline) of $50 \%$ or more relative to baseline and absence of angiomyolipoma progression.

Mutation analysis was completed on the DNA samples of 114 patients participating in EXIST-2 (109 from those with TSC and 5 from those with LAM and angiomyolipomas but not TSC). No mutations were identified in the non-TSC subjects. The proportion of subjects with TSC who had mutations identified and the distribution of mutation types was similar in both the EXIST-2 and EXIST-1 populations (Table 2 and Supplementary Table 2). However, the proportion of patients with TSC1 mutations in EXIST-2 (three patients, $3 \%)$ was much smaller than that in EXIST-1 (13 patients, 13\%) ( $P=0.0178$, Fisher's exact test two-tailed). Furthermore, the observed distribution of TSC1 vs TSC2 mutations in EXIST-2 was significantly different than the aggregate mutation data $\left(P<0.0001, \chi^{2}\right.$ test $) .{ }^{33}$

c SEGA Response by TSC2 Gene Mutation (Large Deletions Only)

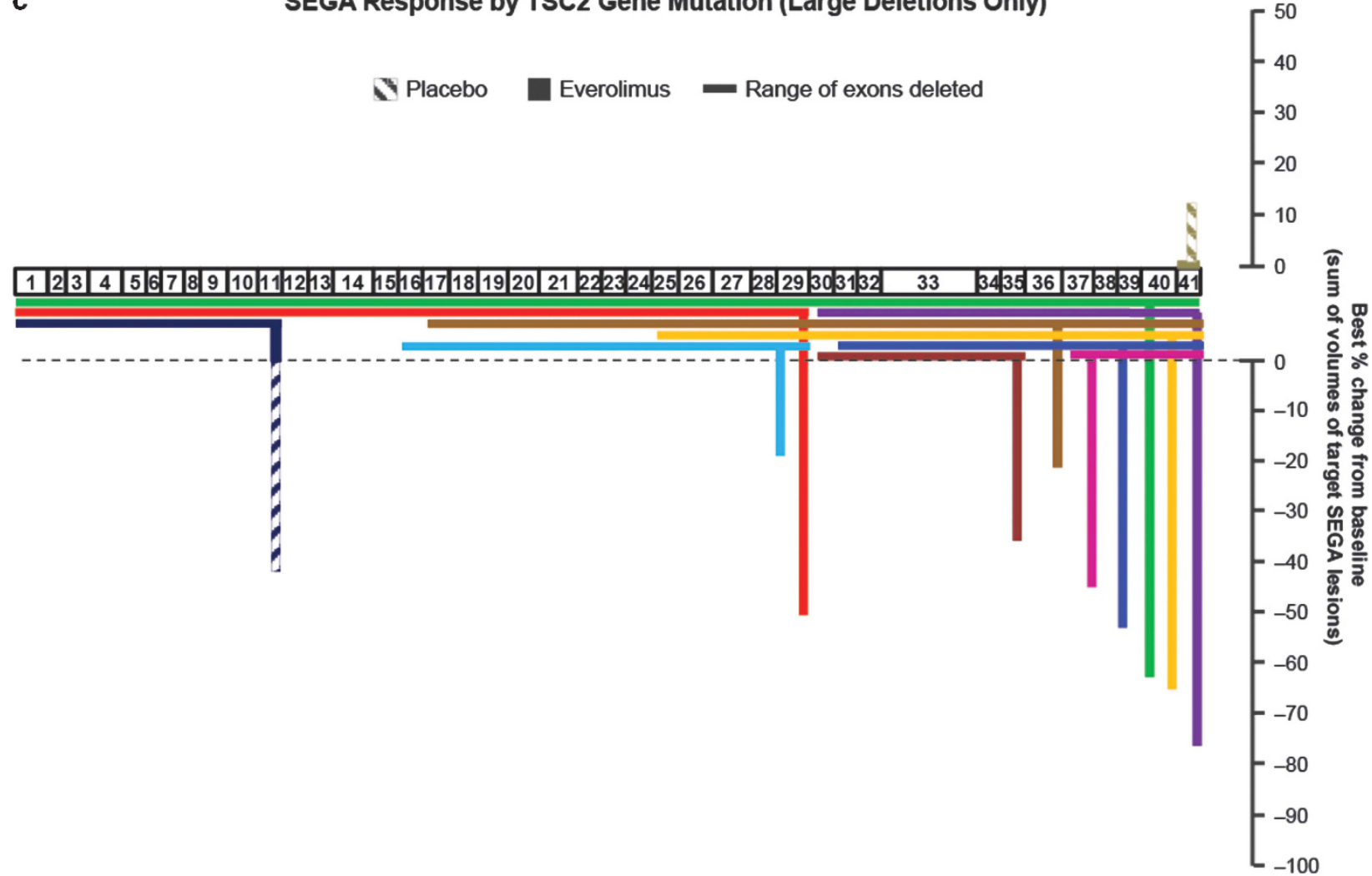

d

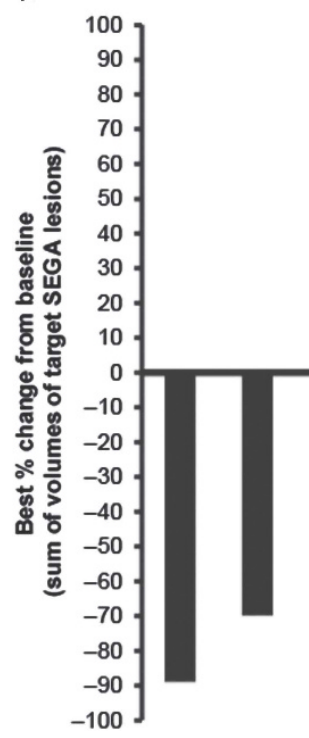

SEGA Response with No Mutation

N Placebo Everolimus

Figure 1 (Continued) 
Correlation between mutation site and type and response to everolimus

The site and type of mutation within TSC1 and TSC 2 compared with response to treatment for both the everolimus- and placebo-treated patients (Figures 1 and 2) were then examined. In both EXIST-1 and EXIST-2, the location of the mutation had no apparent correlation with response. In addition, there was no significant difference in response comparing those subjects with truncating $v s$ nontruncating mutations in either TSC1 or TSC2, those subjects with any TSC2 mutation $v s$ those with no mutation identified or those subjects with any TSC1 mutation $v s$ those with any TSC2 mutation for either EXIST-1 or EXIST-2 analyzed separately (all $P>0.2$, $t$-test).

\section{DISCUSSION}

Multiple previous genotype-phenotype studies have documented that TSC patients with TSC2 mutations have on average more severe disease than those with TSC1 mutations..$^{22,26,31,37}$ A meta-analysis that considered the findings from the three largest series found that subependymal nodules (a precursor lesion to SEGA), intellectual disability, seizures, facial angiofibroma, fibrous forehead plaque, renal angiomyolipoma, renal cysts and retinal phakomata or hamartomas were all significantly more common in individuals with TSC2 mutations than in those with TSC1 mutations. ${ }^{31}$ Diagnosis of renal angiomyolipoma had the highest odds ratio, 8.27 (confidence interval, 4.36-15.7), in TSC patients with TSC2 mutations $v$ s those with TSC1

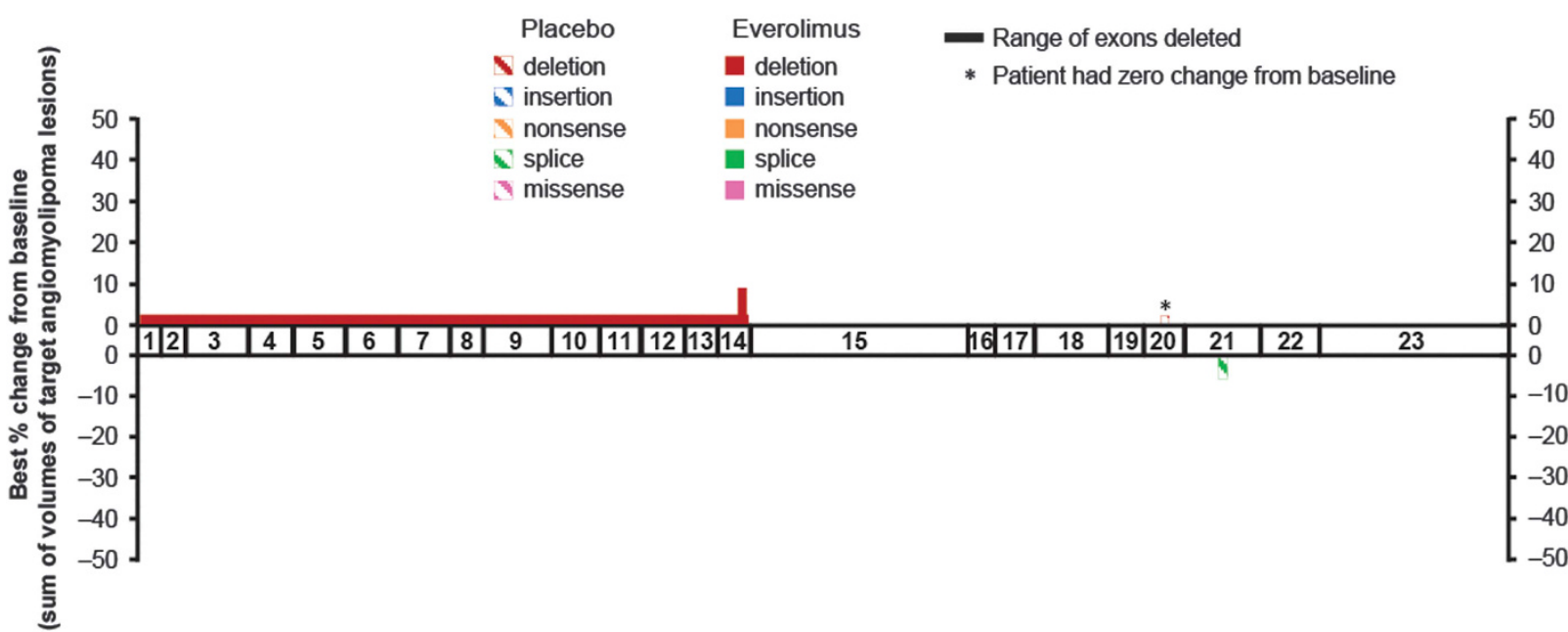

b

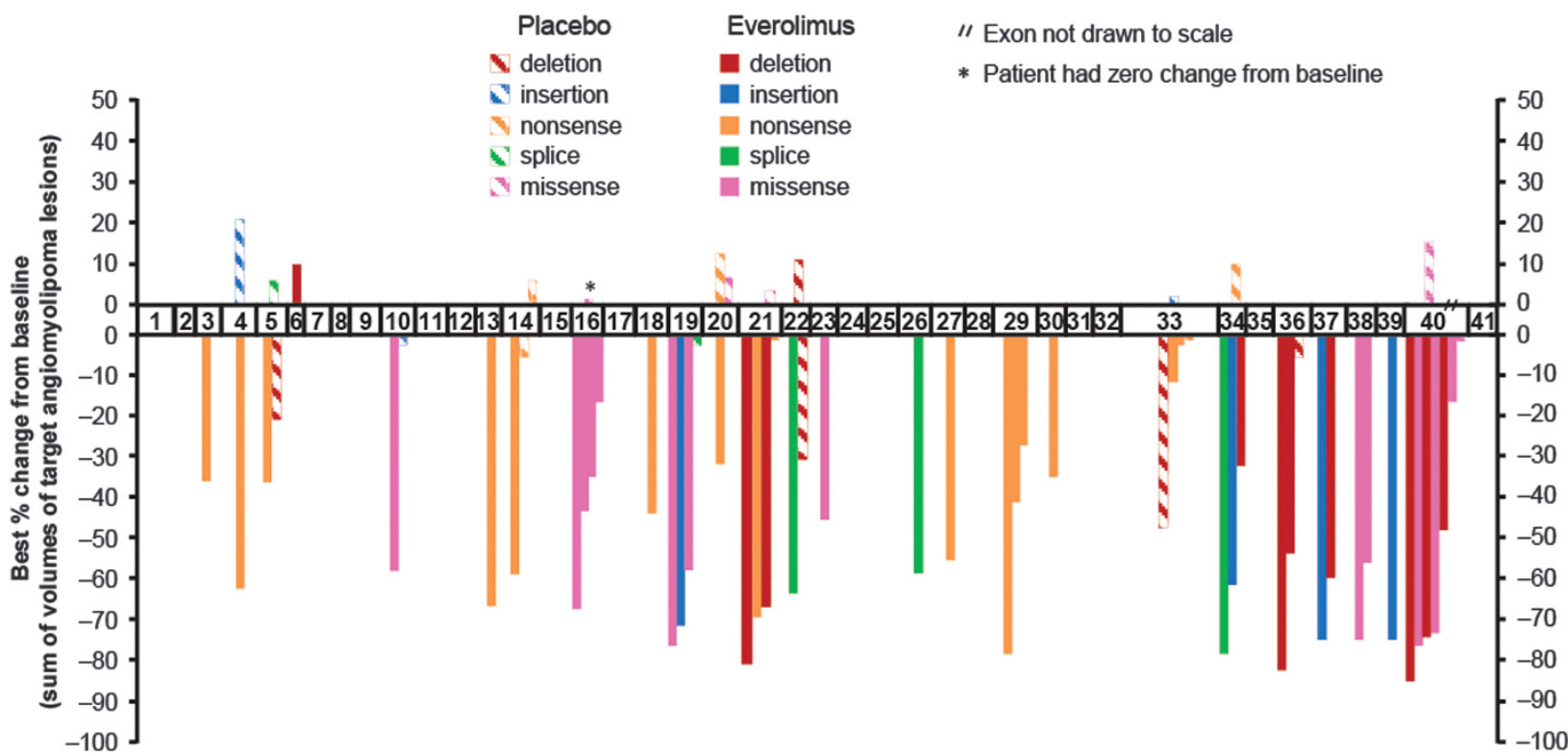

Figure 2 Clinical response to everolimus treatment of angiomyolipomas in EXIST-2 according to mutation type and location in TSC1 and TSC2. The best percentage change in the sum of volumes of target angiomyolipoma lesions is shown on the $y$ axis. The mutation location and type is shown on the $x$ axis, which is a diagram of the exons (drawn proportional to size) of TSC1 (a) or TSC2 (b and $\mathbf{c}$ ). Note that large deletions in TSC1 and TSC2 are indicated by their extent across the exons (a and $\mathbf{c}$ ). Patients without an identified mutation are sorted by response (d). Subjects receiving placebo are shown with striped patterns, whereas those receiving everolimus are shown with solid patterns. 
mutations, of all TSC clinical features considered. ${ }^{31}$ Thus, our findings here that TSC subjects with SEGA enrolled on EXIST-1 had a marginally significant increase in the proportion of TSC2:TSC1 mutations, 87 vs $13 \%(P=0.0662)$, fit these previous observations. Furthermore, the extreme discordance toward TSC2 mutation in the angiomyolipoma TSC patients enrolled in the EXIST-2 trial, 97\% TSC2 vs 3\% TSC1 $(P<0.0001)$, also fits the high odds ratio seen for angiomyolipomas in favor of TSC2 mutations in previous studies. ${ }^{33}$ Clinically, this finding translates to the simple clinical inference that TSC subjects with significant renal angiomyolipoma are much more likely to have a TSC2 mutation than a TSC1 mutation.

Multiple clinical studies have now confirmed the clinical benefit of everolimus and rapamycin for TSC tumors occurring in the brain, kidneys and lungs. $6,7,34,35,38-41$ This includes three randomized clinical trials, including EXIST-1 and EXIST-2, all of which were positive. $^{34,35,40}$ Although several studies have examined the potential for correlation between mutation site and type within TSC1 and TSC2 and various TSC clinical manifestations, few correlations have been seen to date; this is consistent with the model that the majority of mutations in these genes are inactivating, effectively functioning as null alleles. There are two well-documented exceptions in which there is a clear correlation between mutation and clinical phenotype. First, large genomic deletions that affect both TSC2 and the adjacent PKD1 gene lead to early-onset, severe polycystic kidney disease. ${ }^{13}$ Second, there are a number of missense mutations in TSC2 that are associated with a relatively mild phenotype. ${ }^{42-44}$ However, these two types of mutation currently account for $<5 \%$ of reported TSC gene mutations. Seven patients in the EXIST-1 SEGA trial had large genomic deletions extending into the PKD1 gene (Figure 1c). None of the patients in the EXIST-2 angiomyolipoma trial had large genomic deletions extending into the PKD1 gene (Figure 2c). None of the patients on either trial had a missense variant in TSC2 associated with a relatively mild phenotype.

Our observations here that the site and type of mutation in either TSC1 (in the limited number of cases available from each study; Figures $1 \mathrm{a}$ and $2 \mathrm{a}$ ) or TSC2 (Figures $1 \mathrm{~b}, \mathrm{c}$, and $2 \mathrm{~b}$ and $\mathrm{c}$ ) appeared to c

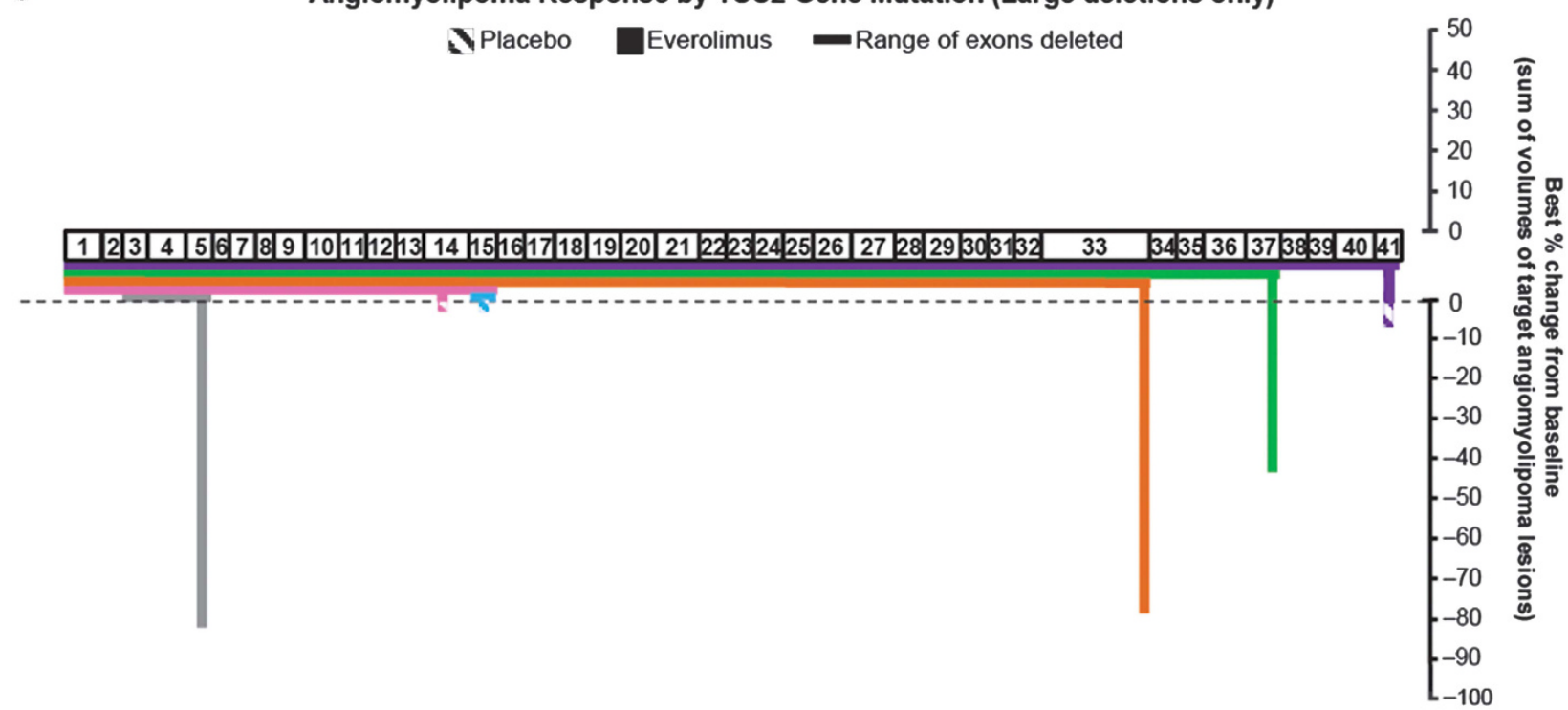

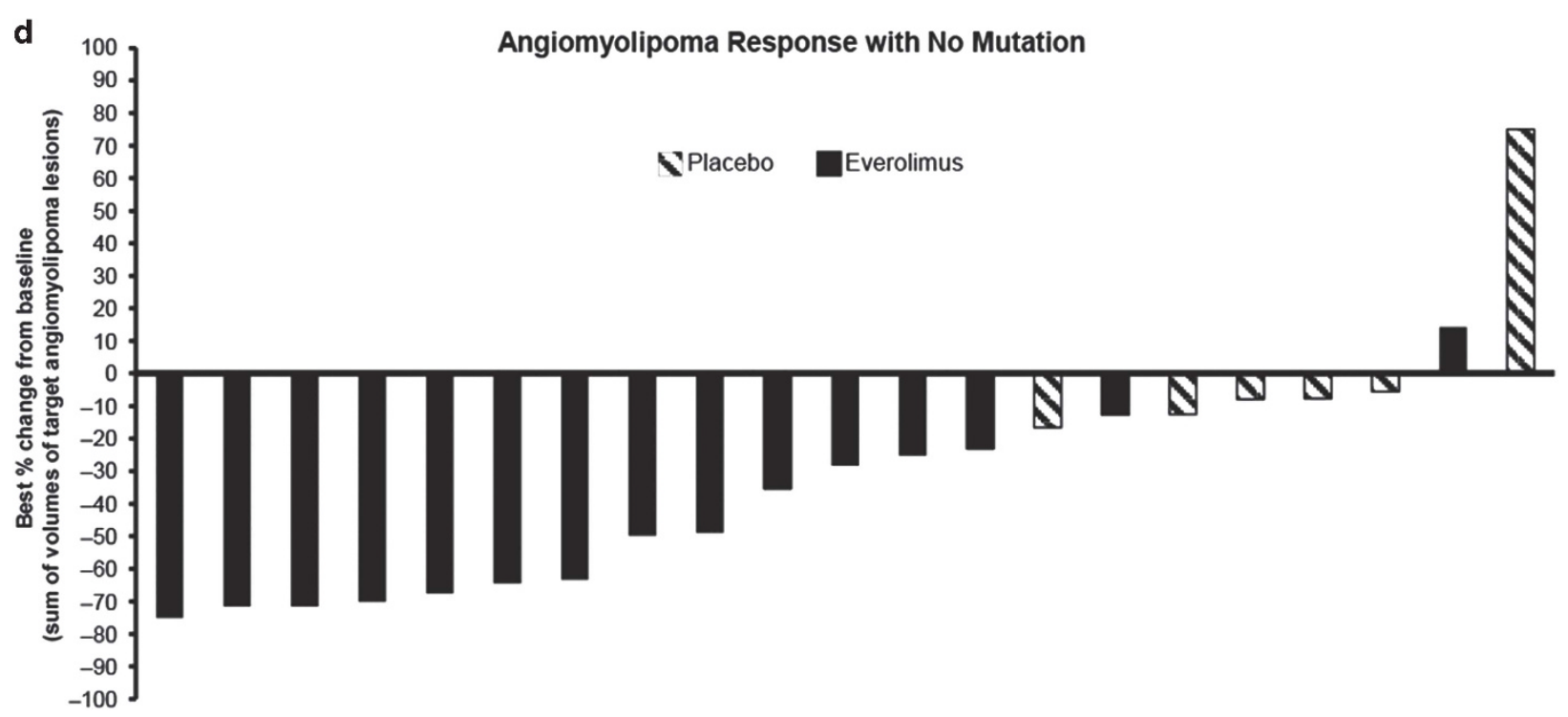

Figure 2 (Continued) 
have no impact on response to everolimus fits with the apparently identical pathophysiological effects of mutations throughout these genes. Nonetheless, the possibility of a correlation was important to examine carefully here because such a correlation might be important for clinical decision making and would also have broader implications for our understanding of the pathogenesis of TSC. The response rate seen in subjects without an identified mutation was similar to that of patients with defined mutations. This observation suggests that subjects without defined mutations have a similar pathogenic mechanism with mammalian target of rapamycin complex 1 activation driving tumor development. This is consistent with the hypothesis that those individuals may have unusual and difficult-to-identify mutations in TSC1 or TSC2, or are mosaic for a TSC1 or TSC2 mutation that was missed by conventional sequencing analysis. ${ }^{45,46}$

\section{CONFLICT OF INTEREST}

DJK has served as consultant to Novartis and received support with the data analysis and writing of this manuscript. SJ has received honoraria from Novartis. JB has received research support from Novartis. DNF has received support for other research at his institution from Novartis; has served as consultant to Novartis (payments to his employer CCHMC); has received honoraria from Novartis and Lundbeck Pharmaceuticals; has been reimbursed by Novartis and Lundbeck Pharmaceuticals for travel costs for lectures; has performed legal work in reviewing medical malpractice cases and occasionally in giving expert testimony for various attorneys. SS has received honoraria from Novartis and Lundbeck Pharmaceuticals. MRP and DC are employees and stock holders of Novartis. JRS has received honoraria and research support from Novartis

\section{ACKNOWLEDGEMENTS}

Novartis Pharmaceuticals Corporation participated in the analysis, writing and review of the manuscript. Editorial assistance was provided by ApotheCom Inc., Yardley, PA, USA, with funding by Novartis Pharmaceuticals Corporation.

1 Kwiatkowski DJ, Thiele EA, Whittemore VH: Tuberous Sclerosis Complex. Weinheim, Germany: Wiley-VCH, 2010.

2 Crino P, Mehta R, Vinters HV: Pathogenesis of TSC in the brain; in: Kwiatkowski DJ, Whittemore VH, Thiele EA (eds): Tuberous Sclerosis Complex: From Genes to Therapeutics. Berlin: Wiley-VCH, 2010, pp 161-185.

3 Franz DN, Krueger DA, Balko MG: Subependymal giant cell astrocytomas; in: Kwiatkowski DJ, Whittemore VH, Thiele EA (eds): Tuberous Sclerosis Complex. Weinheim, Germany: Wiley-VCH, 2010, pp 211-228.

4 Kotulska K, Borkowska J, Roszkowski M et al: Surgical treatment of subependymal giant cell astrocytoma in tuberous sclerosis complex patients. Pediatr Neurol 2014; 50: 307-312.

5 Levine NB, Collins J, Franz DN, Crone KR: Gradual formation of an operative corridor by balloon dilation for resection of subependymal giant cell astrocytomas in children with tuberous sclerosis: specialized minimal access technique of balloon dilation. Minim Invas Neurosurg 2006; 49: 317-320.

6 Franz DN, Leonard J, Tudor $\mathrm{C}$ et al: Rapamycin causes regression of astrocytomas in tuberous sclerosis complex. Ann Neurol 2006; 59: 490-498.

7 Krueger DA, Care MM, Agricola K, Tudor C, Mays M, Franz DN: Everolimus long-term safety and efficacy in subependymal giant cell astrocytoma. Neurology 2013; 80 574-580.

8 Jozwiak S, Nabbout R, Curatolo P, participants of the TSCCMfS, Epilepsy M: Management of subependymal giant cell astrocytoma (SEGA) associated with tuberous sclerosis complex (TSC): Clinical recommendations. Eur J Paediatr Neurol 2013; 17: 348-352.

9 Roth J, Roach ES, Bartels U et al: Subependymal giant cell astrocytoma: diagnosis, screening, and treatment. Recommendations from the International Tuberous Sclerosis Complex Consensus Conference 2012. Pediatr Neurol 2013; 49: 439-444.

10 Dixon BP, Hulbert JC, Bissler JJ: Tuberous sclerosis complex renal disease. Nephron Exp Nephrol 2011; 118: e15-e20.

11 Sooriakumaran $\mathrm{P}$, Gibbs $\mathrm{P}$, Coughlin $\mathrm{G}$ et al: Angiomyolipomata: challenges, solutions, and future prospects based on over 100 cases treated. BJU Int 2010; 105: 101-106.
12 Bissler J, Henske EP: Renal manifestations of tuberous sclerosis complex; in: Kwiatkowski DJ, Whittemore VH, Thiele EA (eds): Tuberous Sclerosis Complex. Weinheim, Germany: Wiley-VCH, 2010, pp 311-326.

13 Sampson JR, Maheshwar MM, Aspinwall R et al: Renal cystic disease in tuberous sclerosis: role of the polycystic kidney disease 1 gene. Am J Hum Genet 1997; 61: 843-851.

14 Au K-S, Rodriguez JA, Finch JL et al: Germ-line mutational analysis of the TSC2 gene in 90 tuberous-sclerosis patients. Am J Hum Genet 1998; 62: 286-294.

15 Young JM, Burley MW, Jeremiah SJ et al: A mutation screen of the TSC1 gene reveals 26 protein truncating mutations and 1 splice site mutation in a panel of 79 tuberous sclerosis patients. Ann Hum Genet 1998; 62: 203-213.

16 Zhang $H$, Nanba E, Yamamoto T et al: Mutational analysis of TSC1 and TSC2 genes in Japanese patients with tuberous sclerosis complex. J Hum Genet 1999; 44: 391-396.

17 Mayer K, Ballhausen W, Rott HD: Mutation screening of the entire coding regions of the TSC1 and the TSC2 gene with the protein truncation test (PTT) identifies frequent splicing defects. Hum Mutat 1999; 14: 401-411.

18 Niida Y, Lawrence-Smith N, Banwell A et al: Analysis of both TSC1 and TSC2 for germline mutations in 126 unrelated patients with tuberous sclerosis. Hum Mutat 1999; 14: 412-422.

19 Jones AC, Shyamsundar MM, Thomas MW et al: Comprehensive mutation analysis of TSC1 and TSC2 and phenotypic correlations in 150 families with tuberous sclerosis. Am J Hum Genet 1999; 64: 1305-1315.

20 van Slegtenhorst M, Verhoef S, Tempelaars A et al: Mutational spectrum of the TSC1 gene in a cohort of 225 tuberous sclerosis complex patients: no evidence for genotypephenotype correlation. J Med Genet 1999; 36: 285-289.

21 Yamashita Y, Ono J, Okada S et al: Analysis of all exons of TSC1 and TSC2 genes for germline mutations in Japanese patients with tuberous sclerosis: report of 10 mutations. Am J Med Genet 2000; 90: 123-126.

22 Dabora SL, Jozwiak S, Franz DN et al: Mutational analysis in a cohort of 224 tuberous sclerosis patients indicates increased severity of tsc2, compared with tsc1, disease in multiple organs. Am J Hum Genet 2001; 68: 64-80.

23 Langkau N, Martin N, Brandt R et al: TSC1 and TSC2 mutations in tuberous sclerosis, the associated phenotypes and a model to explain observed TSC1/TSC2 frequency ratios. Eur J Pediatr 2002; 161: 393-402.

24 Apak A, Haliloglu G, Kose G, Yilmaz E, Anlar B, Aysun S: Mutation analysis of TSC2 gene in 33 Turkish familial cases with tuberous sclerosis. Turk J Pediatr 2003, 45: 1-5.

25 Rendtorff ND, Bjerregaard B, Frodin M et al: Analysis of 65 tuberous sclerosis complex (TSC) patients by TSC2 DGGE, TSC1/TSC2 MLPA, and TSC1 long-range PCR sequencing, and report of 28 novel mutations. Hum Mutat 2005; 26: 374-383.

26 Sancak 0, Nellist M, Goedbloed M et al: Mutational analysis of the TSC1 and TSC2 genes in a diagnostic setting: genotype - phenotype correlations and comparison of diagnostic DNA techniques in Tuberous Sclerosis Complex. Eur J Hum Genet 2005; 13: 731-741.

27 Ali M, Girimaji SC, Markandaya M, Shukla AK, Sacchidanand S, Kumar A: Mutation and polymorphism analysis of TSC1 and TSC2 genes in Indian patients with tuberous sclerosis complex. Acta Neurol Scand 2005; 111: 54-63.

28 Choi JE, Chae JH, Hwang YS, Kim KJ: Mutational analysis of TSC1 and TSC2 in Korean patients with tuberous sclerosis complex. Brain Dev 2006; 28: 440-446.

29 Hung CC, Su YN, Chien SC et al: Molecular and clinical analyses of 84 patients with tuberous sclerosis complex. BMC Med Genet 2006; 7: 72.

30 Kozlowski P, Roberts P, Dabora S et al: Identification of 54 large deletions/duplications in TSC1 and TSC2 using MLPA, and genotype-phenotype correlations. Hum Genet 2007; 121: 389-400.

31 Au KS, Williams AT, Roach ES et al: Genotype/phenotype correlation in 325 individuals referred for a diagnosis of tuberous sclerosis complex in the United States. Genet Med 2007; 9: 88-100.

32 Jansen FE, Braams O, Vincken KL et al: Overlapping neurologic and cognitive phenotypes in patients with TSC1 or TSC2 mutations. Neurology 2008; 70: 908-915.

33 Kwiatkowski DJ: Genetics of tuberous sclerosis complex; in: Kwiatkowski DJ, Whittemore VH, Thiele EA (eds): Tuberous Sclerosis Complex. Weinheim, Germany: Wiley-VCH, 2010, pp 29-60.

34 Franz DN, Belousova E, Sparagana S et al: Efficacy and safety of everolimus for subependymal giant cell astrocytomas associated with tuberous sclerosis complex (EXIST-1): a multicentre, randomised, placebo-controlled phase 3 trial. Lancet 2013; 381: 125-132.

35 Bissler JJ, Kingswood JC, Radzikowska $\mathrm{E}$ et al: Everolimus for angiomyolipoma associated with tuberous sclerosis complex or sporadic lymphangioleiomyomatosis (EXIST-2): a multicentre, randomised, double-blind, placebo-controlled trial. Lancet 2013; 381: 817-824.

36 Henikoff S, Henikoff JG: Amino acid substitution matrices from protein blocks. Proc Natl Acad Sci USA 1992; 89: 10915-10919.

37 Jones AC, Daniells CE, Snell RG et al: Molecular genetic and phenotypic analysis reveals differences between TSC1 and TSC2 associated familial and sporadic tuberous sclerosis. Hum Mol Genet 1997; 6: 2155-2161.

38 Bissler JJ, McCormack FX, Young LR et al: Sirolimus for angiomyolipoma in tuberous sclerosis complex or lymphangioleiomyomatosis. N Engl J Med 2008; 358: $140-151$.

39 Krueger DA, Care MM, Holland $\mathrm{K}$ et al: Everolimus for subependymal giant-cell astrocytomas in tuberous sclerosis. N Engl J Med 2010; 363: 1801-1811.

40 McCormack FX, Inoue Y, Moss J et al: Efficacy and safety of sirolimus in lymphangioleiomyomatosis. N Engl J Med 2011; 364: 1595-1606. 
41 Davies DM, de Vries PJ, Johnson SR et al: Sirolimus therapy for angiomyolipoma in tuberous sclerosis and sporadic lymphangioleiomyomatosis: a phase 2 trial. Clin Cancer Res 2011; 17: 4071-4081.

42 O'Connor SE, Kwiatkowski DJ, Roberts PS, Wollmann RL, Huttenlocher PR: A family with seizures and minor features of tuberous sclerosis and a novel TSC2 mutation. Neurology 2003; 61: 409-412.

43 Wentink M, Nellist M, Hoogeveen-Westerveld $M$ et al: Functional characterization of the TSC2 c.3598C > T (p.R1200W) missense mutation that co-segregates with tuberous sclerosis complex in mildly affected kindreds. Clin Genet 2012; 81: 453-461.

44 Jansen AC, Sancak O, D'Agostino MD et al: Unusually mild tuberous sclerosis phenotype is associated with TSC2 R905Q mutation. Ann Neurol 2006; 60: 528-539.

45 Kwiatkowska J, Wigowska-Sowinska J, Napierala D, Slomski R, Kwiatkowski DJ: Mosaicism in tuberous sclerosis as a potential cause of the failure of molecular diagnosis. N Engl J Med 1999; 340: 703-707.
46 Qin W, Kozlowski P, Taillon BE et al: Ultra deep sequencing detects a low rate of mosaic mutations in tuberous sclerosis complex. Hum Genet 2010; 127: 573-582.

(c) (i) This work is licensed under a Creative Commons Attribution 4.0 International License. The images or other third party material in this article are included in the article's Creative Commons license, unless indicated otherwise in the credit line; if the material is not included under the Creative Commons license, users will need to obtain permission from the license holder to reproduce the material. To view a copy of this license, visit http:// creativecommons.org/licenses/by/4.0/

Supplementary Information accompanies this paper on European Journal of Human Genetics website (http://www.nature.com/ejhg) 